\title{
A simple model for the formation of vegetated dunes
}

\author{
Hiraku Nishimori \\ Hirohisa Tanaka \\ Department of Mathematical Sciences \\ Osaka Prefecture University,Sakai 599-8531,Japan
}

October 23, 2018

\begin{abstract}
A simple model for the dynamics of dunes associated with vegetation is proposed. Using the model, formation processes of transverse dunes, parabolic dunes and elongated parabolic dunes according to two environmental factors: i) the amount of sand at the source,ii)the wind force, are simulated. The results have qualitative correspondence to the real counterpart, and the simplicity of the algorithm and the consequent easiness of the handling of this model provide us with wide applicability for the investigation of the complex interplay between vegetation and dunes.
\end{abstract}

\section{INTRODUCTION}

It is well known that the shape of dunes varies depending on several environmental factors surrounding them, like, the amount of available sand in each desert area, the wind directional variability, the vegetational condition covering sand surface, etc. Among all, the interplay between the dynamics of dunes and the growth of plants remains many aspects unsolved because of the complex nature of the dynamics of the system and the difficulty of the inspection of the theoretical hyposesis through direct observations. Still, many valuable observational studies have been accumulated which provided us detailed environmental conditions at individual arid areas in which typical types of vegetated dunes are seen [1, 2, 3, 4, 5, 6]. One of the pioneering studies among them was made by Hack[2] in which he introduced a phase diagram to show the relation between the wind condition, available sand, the ratio of surface covered by plants, and the dominant type of dunes observed in each arid (or semiarid) area. Here, taking previous observational studies into consideration, we propose a minimal model which realizes the qualitative dynamics of dunes in mildly vegetated arid 
areas. Thereafter, using the model, the formation processes of transverse dunes, parabolic dunes and elongate parabolic dunes according to environmental factors are simulated, that is, the Hack's phase diagram of vegetated dunes is numerically testified. In the below we firstly make a brief explanation of our model and, after that, the outputs obtained through the simulation are shown. Finally we make a discussion on the meaning of the model.

\section{MODEL}

The model is a 2-dimensional(2D) lattice model, wherein two field variables,i)the local height $h(i, j, n)$ of sand bed, and ii)the local density of vegetation $c(i, j, n)$, are allocated at each site of the horizontally extending $N \times N(N=100)$ lattice. Here $\{(i, j) \mid 1 \leq i, j \leq N\}$ and $\{n \mid 0 \leq n\}$ indicate,respectively, horizontal position and time step. Assume wind is constantly blowing in positive $i$ direction, and each $(i, j)$ site covers a sufficiently wider area than that occupied by individual grains. Likewise is the unit time step which reflects a much longer time than individual saltation processes of sand. The above field variables are set to interact each other through the suppression factor $a_{\beta}(i, j, n)$ as explained below. Although many other quantities are considered to contribute to the whole dynamics of the system, we concentrate ourselves on extracting a simple set of relevant factors to investigate the essential dynamics of the whole system. Such methodology for modeling the complex dynamics of dunes with a set of simple rules have recently been introduced by Warner [7] and also by one of present authors[8], with which they succeeded to numerically reproduce the typical types of unvegetated dunes under conditions qualitatively corresponding to the observations made by Wasson and Hyde[9]. More previously, a pioneering model of the formation of vegetated dunes using a minimal set of rules has been introduced by Castro 10 . Although his model is not applicable for the dynamics of dunes without vegetation, neither, does reproduce a wide variety of morphology of dunes in vegetated areas, it successfully simulated a systematic change of the inter-dunes length of vegetated transverse dunes, also the change of their profiles.

Present model is a hybrid model to investigate a wide class of morphodynamics of dunes with vegetation also without vegetation. Here, dynamical rules of sand movement are based on a previous work of the author [8], whereas the rules for time evolution of plants have many similarities to the model by Castro 10 .

I. For the evolution of $c(i, j, n)$, the local density of plants, we adopt a set of simple rules. In an extreme case where the height of sand surface remains unchanged with time, wherein plants can grow thick up to the saturation density without being cut away by the drastic deflation of ground or being buried by the rapid accumulation of sands, $c(i, j, n)$ is allowed to increase linearly until the maximum value $c_{\max }$. On the other hand, if the temporal change of surface height is too fast, the growth rate of plants is suppressed or some of them may wither up and die, then $c(i, j, n)$ decrease down to the minimum limit $c_{\text {min }}$. To reflect the above situation, we use a discrete dynamics which is a sectional linear map as shown in 
fig.1. Specifically, the dynamics is expressed as,

$$
\begin{aligned}
& c(i, j, n+1)=A(c(i, j, n)-b(i, j, n))+c_{\operatorname{mim}} \quad\left(b \leq c \leq\left(c_{\max }-c_{\min }\right) / A+b\right) \\
& c(i, j, n+1)=c_{\max } \quad\left(\left(c_{\max }-c_{\min }\right) / A+b<c\right) \\
& c(i, j, n+1)=c_{\operatorname{mim}} \quad(c<b)
\end{aligned}
$$

here $b \equiv b(i, j, n) \equiv|h(i, j, n)-h(i, j, n-1)|, c \equiv c(i, j, n)$ and $A$ is a constant to determine the growth rate of plants.

II. for the evolution of $h(i, j, n)$, discretized conservation law of $h(i, j, n)$,

$$
h(i, j, n+1)-h(i, j, n)=Q_{\text {in }}(i, j, n)-Q_{\text {out }}(i, j, n)
$$

holds, where $Q_{i n}(i, j, n)$ is the total mass of sand coming into site $(i, j)$ at time step $n$, while $Q_{\text {out }}(i, j, n)$ is the same quality leaving from $(i, j)$ at $n$. Both of the saltation flux and the creep flux contribute to $Q_{i n}(i, j, n)$ and $Q_{\text {out }}(i, j, n)$. Specifically the saltation flux caused by the grains leaving from $(i, j)$ at $n$ is expressed by the production of its mass $m$ and length $l$ like,

$$
q_{\text {sal }}(i, j, n)=q_{0}(\tanh (\nabla h(i, j, n))+1.0)(\tanh (-\nabla h(i, j, n))+1.0+\alpha) .
$$

where $\nabla h(i, j, n)$ means $h(i, j, n)-h(i-1, j, n)$ and $\alpha$ is a constant to determine the bed-load in the windward slope of dunes. The above reflects the qualitative nature of wind, also the resulting saltation flux around dunes which is intensive in the windward particularly around the crest, whereas almost no flux in the lee side[8, 11](fig.1(b)). On the other hand, the flux by creep $q_{\text {creep }}(i, j, n)$ is set proportional to the local gradient of sand surface. Although this may be a very crude approximation, it has some sense for a qualitative description of the morphodynamics of dunes as discussed afterward. The crucial effect caused by permitting the growth of plants is such that the sand transport sharply decreases when the cover ratio of sand surface by plants exceeds a critical value. To realize the situation in a simple expression, the suppression factor $a_{\beta}(i, j, n)$ is introduced like,

$$
a_{\beta}(i, j, n)=1+\beta\left(\tanh \left(c-c_{c r}\right)-1\right) . \quad(0 \leq \beta \leq 1 / 2)
$$

Here, $c_{c r}$ is the critical vegetation density over which the movement of sand sharply decreases, and $\beta$ determines the maximum efficiency of suppression(fig.1(c)). The value of $\beta$ depends on whether it is for the saltation flux or for the creep flux. With the suppression factor, the saltation/creep flux is forced to decrease as $q(i, j, n)=a_{\beta}(i, j, n)^{2} q^{\prime}(i, j, n)$ where $q(i, j, n)$ and $q^{\prime}(i, j, n)$ are, respectively, the local flux by saltation/creep with vegetation and without vegetation,.

\section{RESULTS}

Intending to compare the simulation outputs with the diagram by Hack, two kind of quantities are chosen as the control parameters; One is i)the amount of 
sand at the source. Namely, the average height, $\left\langle h_{\text {source }}\right\rangle$, of the sand surface at the source sites, $\{(i, j) \mid i=1,2 \quad 1 \leq j \leq N\}$. Specifically, uniformly random numbers between $2<h_{\text {source }}>$ and 0 are allocated on these sites at each time step. The other control parameter is ii)the wind strength which should be a monotonically increasing function of saltation flux. Specifically, the variable $q_{0}$ in eq.(2) is adopted as the index of wind strength. Note that the vegetation density, which is one of the axes in the diagram by Hack, is not adopted as a control parameter because it is rather a resultantly attained quantity after the above two control parameters are fixed. Simulations are initiated from flat sand surface except the source area, namely, $h(i, j, 0)=0$ holds except the source sites, while the initial vegetation density $c(i, j, 0)$ at each site is set random around the average value $\langle c(i, j, 0)\rangle$ which is between $c_{\max }$ and $c_{\min }$. Note the boundary condition in $j$ direction, which is perpendicular to the wind direction, is set periodic and the leeward boundary in $i$ direction is set as the free boundary. Below the initial level 0 of sand surface, erosion of sand surface is inhibited to realize the existence of the hard ground or the ground water table. Using these rules, spontaneous formation processes of typical types of dunes are observed.

The results are; First of all, when the wind force is too weak, regardless of the amount of sand supply at the source, clearly shaped dune will not appear in the system(fig.2(a)). It is also the cases where the amount of sand supply is too small. On the other hand, with more than a certain strength of wind force and a certain amount of sand supply, two types of clearly shaped active dunes are formed;

i) When sufficiently large amount of sand is supplied under rather strong wind, transverse dunes, barchan dunes or both of them will dominate in the system. In more detail, small parabolic dunes formed just lee of the sand source soon develop into barchan dunes, which connect each other to grow larger as they move, to form transverse dunes the crests of them extend, roughly, perpendicular to the direction of wind(fig.2(b)).

ii)If the amount of supplied sand or the wind force is slightly less than the above, parabolic dunes will prevail in the system(fig.2(c)(d)). They have arms extending to the windward direction. At the center of each parabolic dune, hollow is developed in which surface erosion proceeds down to the level of hard ground to serves the dune's nose with sand. There we can see the inclination that the length of the arms varies depending on the wind force, namely, the arms extend the longer under the stronger wind. Especially in the case with rather small amount of sand, elongate parabolic dunes [4] with the shapes like hair-pins will appear(fig.2(d)). The arms of such elongate dunes, if without their noses, seem like pairs of linear dunes.

\section{DISCUSSION}

The above results are summarized in a phase diagram as shown in fig.4. This diagram is not directly compared with that of Hack because of the less number of axes in our diagram. Also, in the diagram, ambiguous area is left in which 
condition irregular mounds of sand are formed, which are not easily categorized into other dominant types of dunes. Still, it has qualitatively good correspondence to the previous observational studies including that of Hack. Especially, the systematic change of the dunes' morphology from the transverse(or barchan) dunes to the parabolic or the elongate parabolic dunes according to the amount of available sand (or to the wind force), is clearly simulated. Moreover no dune formation is realized under too weak wind nor is under too small amount of sand supply. The results indicate that this simple model contains intrinsic dynamics by which the morphodynamics of the vegetated dunes is decisively affected, and that through this model theoretical hypotheses on the formation dynamics of vegetated dunes can be testified.

On the other hand, present study leaves several, possibly crucial, situations untreated. Firstly, the wind is always blowing in one direction. This condition seems very hard for the spontaneous growth of linear dunes considering the cases of unvegetated dunes. Consequently, straightly extending sand dunes/ridges appear only in behind parabolic dunes as the traces of their arms. This is one of the possible origin of vegetated linear dunes 4 . But it seems not the general scenario for the formation of linear dunes, thus, the study under complicated wind regimes is one the next issues. Secondly, the existence of the angle of repose was not explicitly incorporated in the model. Here, instead, all the dynamics along sand surface was modeled by the diffusion-like creep process. Of course such a rough simplification can cause the imperfect reproduction of the real counterparts, like inhomogeneous angle of slope at the lee face of a dune unlike the actual slip face. On the problem, in the previous studied, we demonstrated that even such a simplified modeling can work effectively, at least for the investigation of macroscopic morphodynamics of unvegetated dunes, like, simulating distinguishable barchan, seif, star and other types of dunes. Also in the present simulations with vegetation, distinguishable parabolic and other types of dunes successfully appeared according to the corresponding situations in the semiarid desert areas. However, for the investigation of more quantitative aspects of the system, the explicit introduction of the angle of repose into the model is required, and it also be an important step to the inclusive understanding of the system.

Notwithstanding all, we believe the present study using such a minimal model is one of the effective way to understand the complex interplay between dunes and vegetation. Systematic search of complex systems using such bold simplifications would, more or less work as the complementary way which fill the big blank between the observational studies of such systems and the, otherwise hardly testified, theoretical hyposesis.

\section{ACKNOWLEDGEMENTS}

This research was supported by the Grant-in-Aid for Scientific Research of JSPS (c11837017). 


\section{References}

[1] Cook,R.,Warren,A., and Goudie, A., 1993, Desert Geomorphology: London, UCL press.

[2] Hack,J.T., 1941, Dunes of the western Navajo Country: Geographical Review, v.31, p.240-263.

[3] Thomas,D.S.G., and Tsoar,H., 1990, The geomorphological role of vegetation in desert dune systems: Vegetation and Erosion, p.471-489.

[4] Pye,K., 1982, Morphological development of coastal dunes in a humid tropical environment, cape bedford and cape flattery, North Queensland: Geografiska Annaler, 64A, p.213-227.

[5] Tsoar,H., and Moller,J-T., 1982, Aeorian Geomorphology:Boston, Allen and Unwin, p.75-97.

[6] Halsey,L.A., Catto,N.R., and Rutter,N.W., 1990, Sedimentology and development of parabolic dunes, Grande Prairie dune field, Alberta: Canadian Journal of Earth Sciences, v.27, p.1762-1772.

[7] Werner,B.T., 1995, Eolian dunes: Computer simulations and attractor interpretation:Geology. v.23, p.1107-1110.

[8] Nishimori, H., Yamasaki, M., and Anderson, K. H., 1998, A simple model for the various pattern dynamics of dunes: International Journal of Modern Physics, v.12, p.257-272.

[9] Wasson,R.J., and Hyde,R., 1983, Factors determining desert dune type :Nature,v.304, p.337-339.

[10] de Castro,F., 1995, Computer simulation of the dynamics of a dune system: Ecological Modelling, v.78, p.205-217.

[11] Rassmussen,K., 1989, Proc of Symposium 'coastal sand dunes':Edinburg, Royal Society of Edinburg, B96, p.129-147. 


\section{Figure Captions}

Fig.1 Schematic explanations of the present model; (a-I)The Map $c(i, j, n) \rightarrow$ $c(i, j, n+1)$ to describe the discretized time evolution of plants density. Without the temporal change $b(i, j, n)$ of surface height, plants density monotonically increases up to the saturation value $c_{\max }$. (a-II)With more than a certain speed of surface rise or deflation, plants at the surface are, more or less, damaged because they are buried or cut away, then, $c(i, j, n)$ will decrease with time with the lower limit $c_{\text {min }}$. b)Local sand flux by saltation $q_{\text {sal }}$ of eq.(2) is given as a function of local slope $\nabla h$ of sand surface in the direction of wind, which reflects the observational fact, namely, large bed load in the windward particularly around the crest(indicated by the arrow), whereas sharply it decreases in the lee side [8, 11]. c)Suppression factor $a$ of sand flux as a function of local density of vegetation $c$. Above a critical density $c_{c r}$ of vegetation, the flux of sand drastically decreases as described in eq.(3).

Fig.2 Snapshots of simulated dunes under various pairs of control parameters, wind force and sand supply. The left part in each figure shows the spatial distribution of vegetation density $d(i, j, n)$. The darker tone indicates the more densely vegetated place, whereas white parts indicates the areas with bare sand surfaces. The right part in each figure shows the surface height distribution $h(i, j . n)$, where the darker position means the higher surface. Sand is supplied from the most windward 2 rows. Steady wind is blowing from the left to the right. (a)When the wind force is too weak no distinguishable dune is formed. It is also the case for too small amount of sand supply at the source. (b)Under rather strong wind with sufficient amount of sand supply transverse dunes prevail while small parabolic dunes are seen just lee of the sand source. The latter will soon grow up to the former. (c)Parabolic dunes, the arms of which extend in the windward direction, are formed under mildly blowing wind with intermediate amount of sand at the source. (d)If the amount of sand is comparatively small within this regime, thin and long parabolic dunes, namely, elongate parabolic dunes will grow. They look like rather linear dunes if without their noses.

Fig.3 Phase diagram to show the dominant types of dunes under various pairs of control parameters. The alphabets in the diagram indicate the conditions corresponding to respective snapshots in fig.2. Symbols $\triangle \mathrm{s}$ and $\bullet$ s indicate, respectively, parabolic dunes, and, barchan(or transverse) dunes, whereas $\times \mathrm{s}$ mean the conditions for no dune formation. The $\triangle \mathrm{s}$ accompanied by ${ }^{*}$ mean the conditions for the development of rather thin parabolic dunes with long arms. At the condition with the symble $\square$, many irregular mounds are formed which are not clearly categorized as particular type of dunes. 


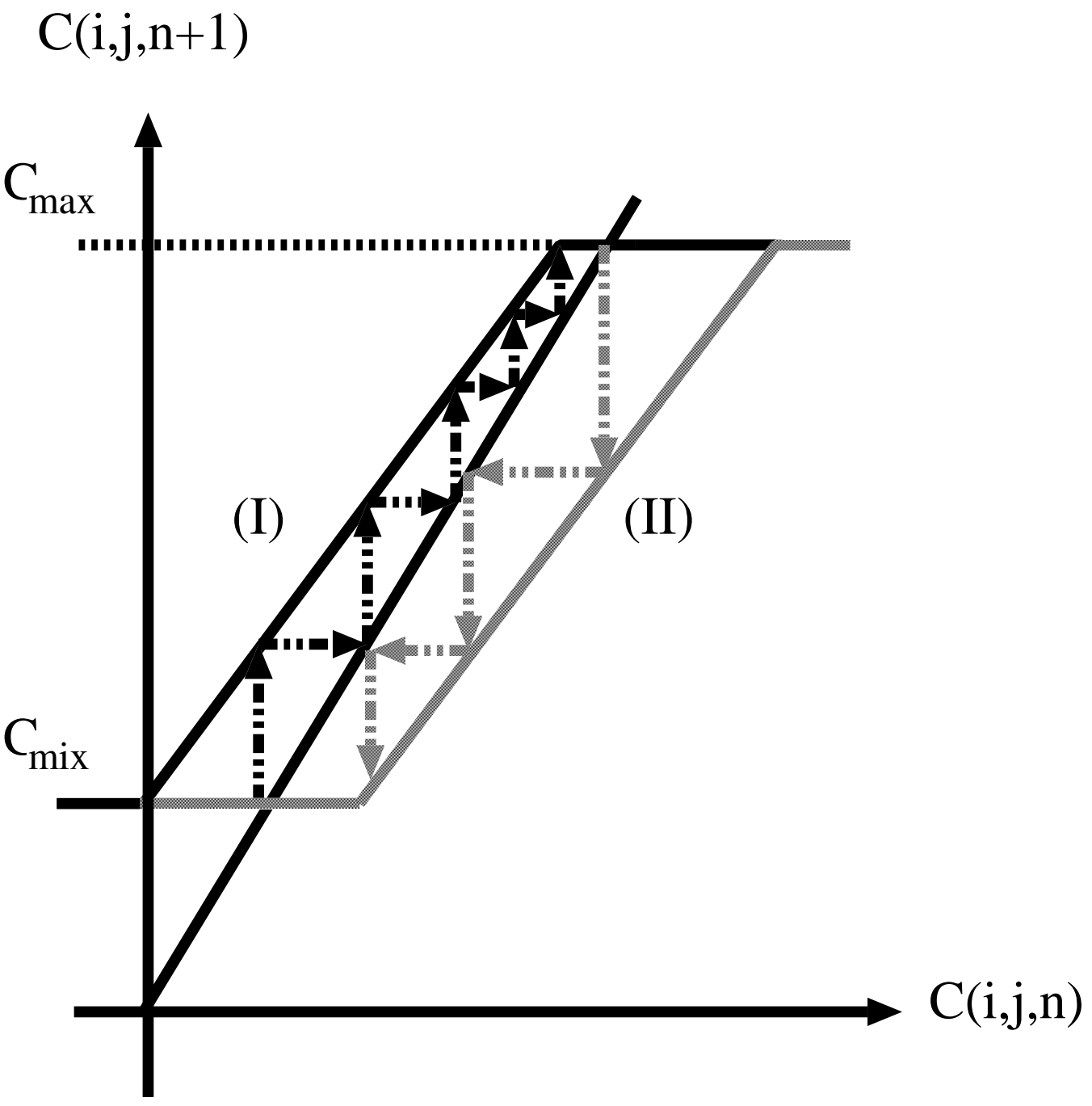



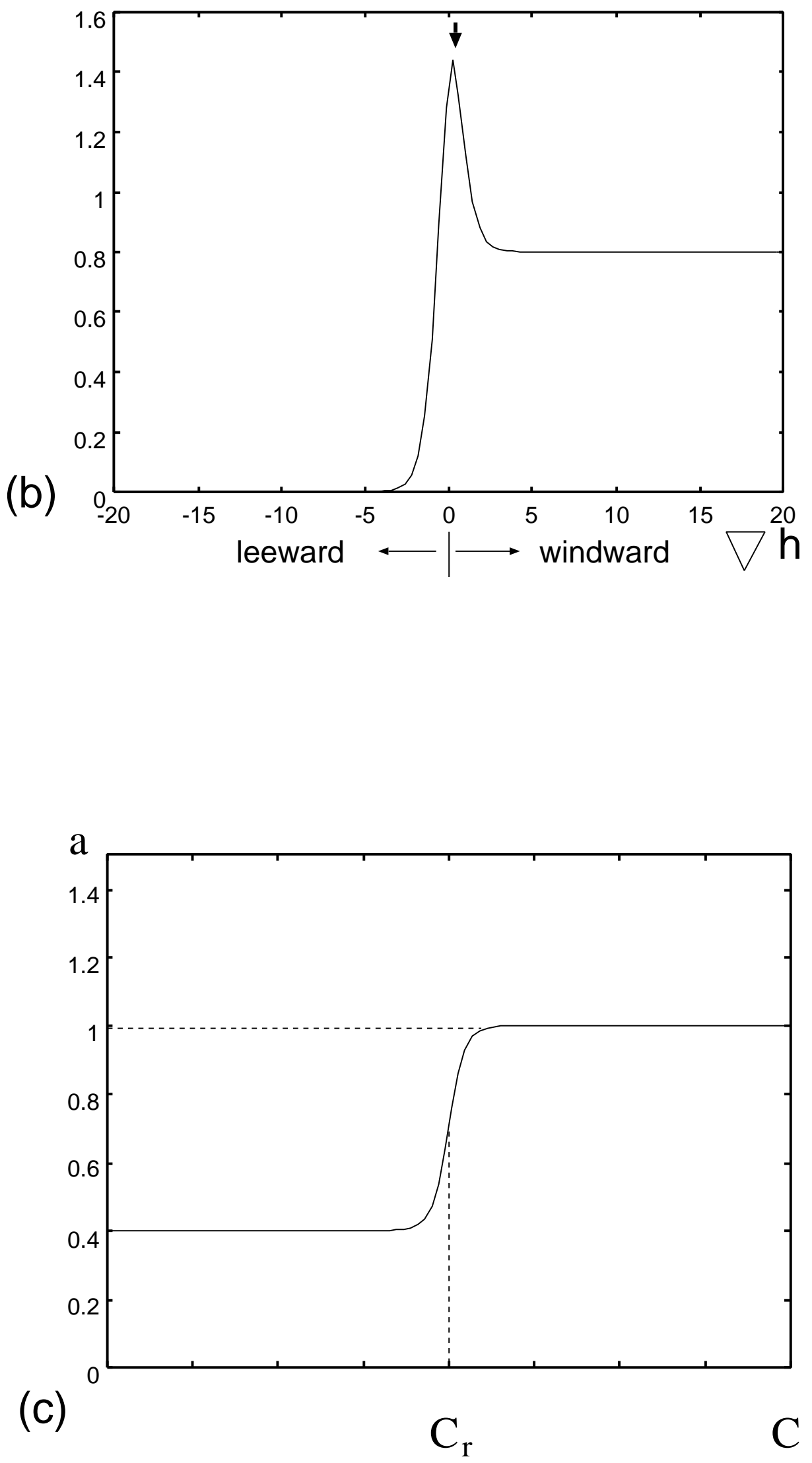


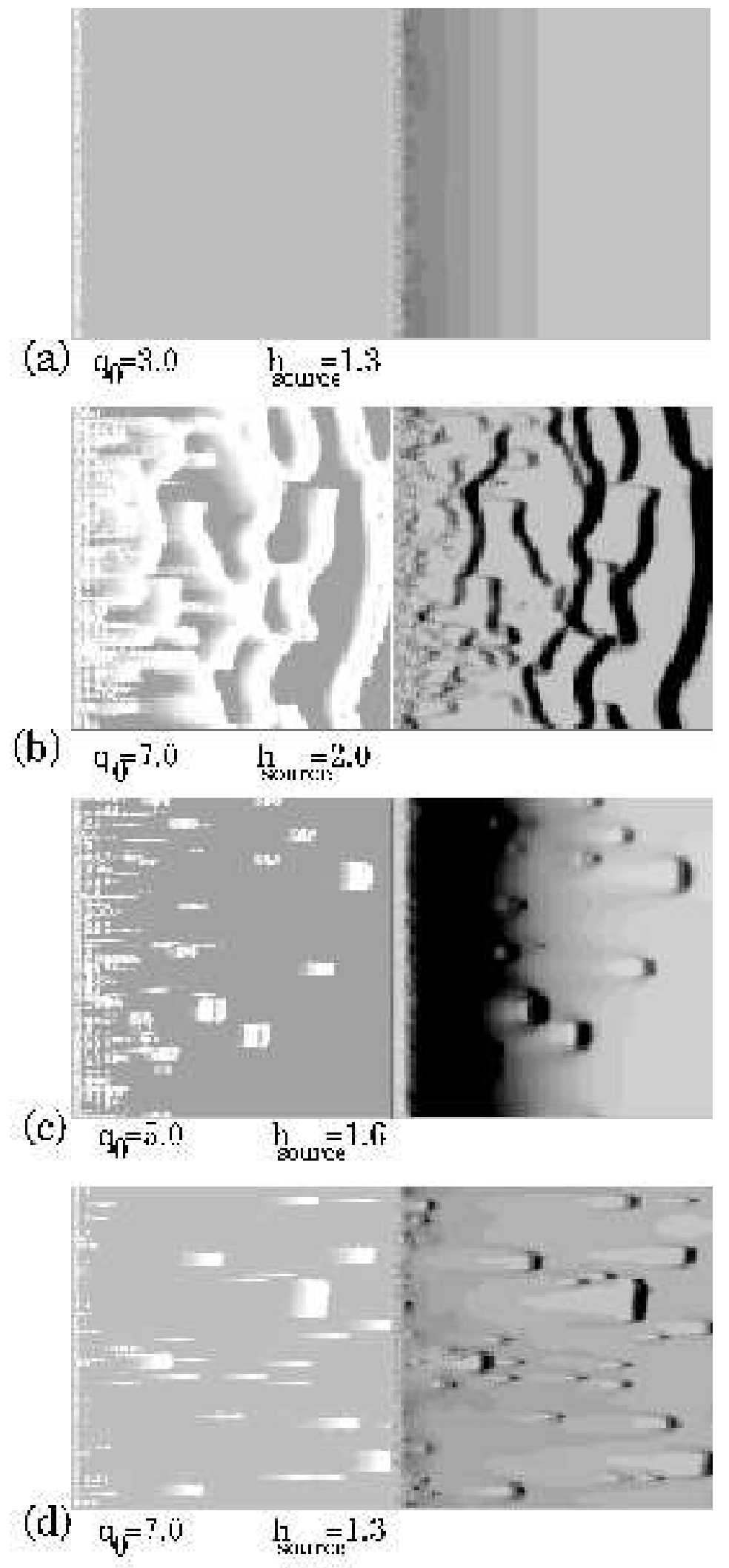




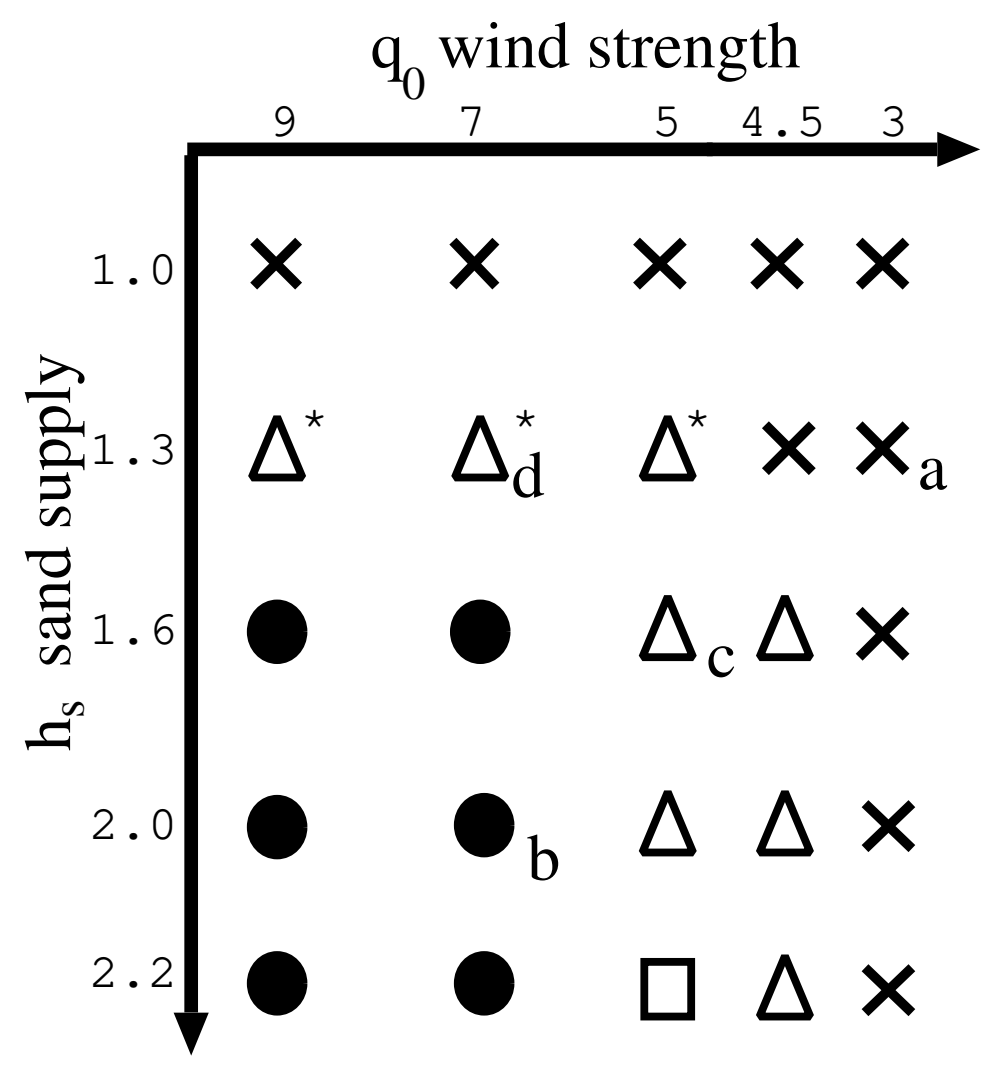

Fig.3 H.Nishimori \& H.Tanaka 